\title{
UNIBRANCHED PRIME IDEALS AND GOING DOWN IN PI RINGS
}

\author{
PHILLIP LESTMANN
}

\begin{abstract}
The purpose of this paper is to answer the question of whether going down is equivalent to unibranchedness of prime ideals in integral extensions of prime PI rings. We show by example that, in general, the answer is no; and we find an additional condition which, together with going down, implies prime ideals of ht $>1$ are unibranched.
\end{abstract}

In [4] McAdam proved the following theorem: Let $R$ be a commutative Noetherian domain and let $T$ be a domain between $R$ and its integral closure. Then $R \subset T$ has going down if and only if every prime $P$ of $R$ with rank (ht) $P>1$ is unibranched in $T$.

In this paper we regard a Noetherian prime PI ring as being the (possibly) noncommutative analogue to a commutative Noetherian domain; its central integral closure as defined in [7] as the analogue of integral closure; and ask whether the conclusion of the theorem holds in this case. We show that an additional condition to going down is necessary to imply unibranchedness in the noncommutative case and prove a generalization of the above theorem using this condition. Two counterexamples are given which show that the conditions are also necessary.

By a PI ring we understand an associative ring which satisfies a polynomial identity. Standard results on PI rings may be found in [6]. The usual definitions for lying over (LO), going up (GU), incomparability (INC), and going down (GD) are assumed here. They may be found in [3].

We list below some key definitions.

Definition 1. For any two rings $R \subset S$, let $S^{R}=\{s \in S \mid s r=r s$ for all $r \in$ $R$ \}. We say that $R \subset S$ is an extension if $S=R S^{R}$. The extension is central if $S=R Z(S)$ where $Z(S)=\{s \in S \mid t s=s t$ for all $t \in S\}$ (the center of $S$ ).

Definition 2. Let $R$ be a prime PI ring, $Q$ its ring of quotients, and $K=Z(Q)$. Then the central integral closure of $R$ is obtained by adjoining to $R$ all elements of $K$ ( = the quotient field of $Z(R)$ ) which satisfy a monic polynomial with coefficients in $R$.

Definition 3. Let $R$ be a prime ring; i.e., (0) is a prime ideal. A prime ideal $P$ of $R$ is said to have height 1 (ht 1$)$ if $P_{1} \subset P, P_{1}$ a prime ideal of $R$, implies $P_{1}=(0)$ or $P_{1}=P$. If $P$ properly contains a ht 1 prime, we will say $P$ has ht $>1$.

Received by the editors April 18, 1980.

AMS (MOS) subject classifications (1970). Primary 16A12, 16A38, 16A56; Secondary 13 B20.

Key words and phrases. Integral extension, integral closure, going down, prime ideal, PI ring, Noetherian, unibranched. 
Definition 4. Let $R \subset S$ be an extension having LO.

(i) If a prime ideal $P$ of $R$ is such that for prime ideals $Q$ and $Q_{1}$ of $S$, $Q \cap R=P$ and $Q_{1} \cap R=P$ forces $Q=Q_{1}, P$ is said to be unibranched in $S$.

(ii) If $P$ is a prime ideal of $R$ so that whenever primes $Q$ and $Q_{1}$ of $S$ have $Q \cap R=P=Q_{1} \cap R$ and $Q \cap Z(S)=Q_{1} \cap Z(S)$ it must be that $Q=Q_{1}$, then $P$ will be called weakly unibranched in $S$.

Observe that unibranched always implies weakly unibranched. In the case of commutative rings all prime ideals are weakly unibranched. Example 1 shows that weakly unibranched does not imply unibranched in general. Example 2, given after the Theorem below, exhibits a central integral extension having GD in which the small ring has a prime ideal that is not weakly unibranched.

EXAMPLE 1. Let $J=$ the integers, $S=J[i]=\left\{a+b i \mid i^{2}=-1 ; a, b \in J\right\}$, and $R=J+5 i J=\{a+5 b i \mid a, b \in J\}$. Then $5 J+5 i J$ is a prime ideal of $R$ having two primes of $S$ lying over it. The two primes are $(2+i) S$ and $(2-i) S$. Note that $S$ is the integral closure of $R$ in its quotient field. Both rings are Noetherian.

LEMMA. Let $R$ be a Noetherian prime PI ring and $Q_{1}$ and $Q_{2}$ two prime ideals of $R$ of $\mathrm{ht}>1$ for which $Q_{1} \cap Z(R) \not Q_{2} \cap Z(R)$. Then there are infinitely many primes $P$ of $R$ properly contained in $Q_{1}$ such that $P \not \subset Q_{2}$ and ht $P=1$.

Proof. Let $T R$ be the central extension of $R$ obtained by adjoining to $R$ all coefficients of characteristic polynomials of elements of $R$ (which coefficients lie in the quotient ring of $R$ ). By Theorem 7 of [1] $T R$ is also Noetherian. By Lemma 1 and Theorem 2 of [7] the extension is integral; hence GU, LO, and INC hold by Theorem 1 of the same paper. It follows that there exists a prime ideal $Q_{1}^{\prime}$ of $T R$ such that $Q_{1}^{\prime} \cap R=Q_{1}$ and ht $Q_{1}^{\prime}>1$. Let $Q_{21}^{\prime}, Q_{22}^{\prime}, \ldots, Q_{2 k}^{\prime}$ be the set of primes of $T R$ which lie over $Q_{2}$ (necessarily of finite number since $T R$ is Noetherian); and let $A=Q_{21}^{\prime} \cup Q_{22}^{\prime} \cup \cdots \cup Q_{2 k}^{\prime}$. Since $Q_{1} \cap Z(R) \not \subset Q_{2 j}^{\prime}, 1<j<k$, it follows that $Q_{1}^{\prime} \cap Z(T R) \not Q_{2 j}^{\prime} \cap Z(T R)$. Let $q_{1}, q_{2}, \ldots, q_{n}$ be any finite collection of ht 1 primes of $T R$ lying inside $Q_{1}^{\prime}$ but not in $A$. $T R$ is integral over $Z(T R)$ [1] and $q_{i} \neq Q_{1}^{\prime}$; so $q_{i} \cap Z(T R) \neq Q_{1}^{\prime} \cap Z(T R), \quad 1<i<n$. Then $Q_{1}^{\prime} \cap Z(T R) \not q_{1}$ $\cup \cdots \cup q_{n} \cup A[5]$.

Now for any $a \in Q_{1}^{\prime} \cap Z(T R) \backslash\left(A \cup q_{1} \cup \cdots \cup q_{n}\right)$ there is a ht 1 prime $q$ of $T R$ such that $a \in q \subset Q_{1}^{\prime}$ [2]. Obviously, $q \neq q_{i}, 1<i<n$. Therefore no finite collection of ht 1 primes of $T R$ which lie inside $Q_{1}^{\prime}$ but outside $A$ can exhaust the collection $\left\{q_{r}\right\}$ of such primes. For each $r, q_{r} \cap R$ is a prime ideal of $R$ properly contained in $Q_{1}$ by INC. If $q_{r} \cap R \subset Q_{2}$, then $q_{r} \subset Q_{2 j}^{\prime}$ for some $j$ by GU. This would contradict the assumption that $q_{r} \not \subset A$. Thus to finish the Lemma, we need only show that at most finitely many of the ideals $q_{r} \cap R$ can have ht $>1$.

Consider in $R$ the ideal $I=\{r \in R \mid r T R \subset R\}$. Since $T R$ is finitely generated as an $R$-module by elements of the quotient field of $Z(R), I \neq(0)$. It is not hard to see that a prime ideal of $R$ not containing all of $I$ must be unibranched in $T R$. Since the primes $\left\{q_{r}\right\}$ have ht 1 in the Noetherian ring $T R$, at most finitely many can contain $I$. Hence, at most a finite number of the primes $q_{r} \cap R$ can contain $I$. A simple application of GU and LO shows that $I \not \subset q_{r} \cap R \operatorname{implies} \operatorname{ht}\left(q_{r} \cap R\right)=$ 1. Thus the Lemma is proved. 
THeOREM. Let $R \subset R^{\prime}$ be an extension of rings where $R$ is a Noetherian prime PI ring and $R^{\prime}$ lies in the central integral closure of $R$. Then $R \subset R^{\prime}$ has GD and prime ideals of $R$ of ht $>1$ are weakly unibranched in $R^{\prime}$ if and only if prime ideals of $R$ of ht $>1$ are unibranched in $R^{\prime}$.

PROof. If primes of $R$ of ht $>1$ are unibranched in $R^{\prime}$, it is trivial that $R \subset R^{\prime}$ has GD since it has LO and GU [7]. Also, as was pointed out above, unibranchedness always implies weak unibranchedness.

In the other direction, suppose that $R \subset R^{\prime}$ has GD and that ht $>1$ primes of $R$ are weakly unibranched in $R^{\prime}$. Let $Q_{1}$ and $Q_{2}$ be two primes of $R^{\prime}$ chosen such that $Q_{1}$ and $Q_{2}$ lie over a given prime ideal $P$ of $R$ where ht $P>1$. Note that GD forces both $Q_{1}$ and $Q_{2}$ to be of ht $>1$. By hypothesis $Q_{1} \cap Z\left(R^{\prime}\right)=Q_{2} \cap Z\left(R^{\prime}\right)$ would imply $Q_{1}=Q_{2}$. We thus assume that $Q_{1} \cap Z\left(R^{\prime}\right) \not \subset Q_{2} \cap Z\left(R^{\prime}\right)$.

Choose $a \in Q_{1} \cap Z\left(R^{\prime}\right) \backslash Q_{2}$ and let $S=R[a]$. Since $a$ is integral over $R, S$ is a finite $R$-module and hence Noetherian. There are only finitely many ht 1 primes of $S$ which contain the ideal $I=\{r \in R \mid r S \subset R\}$. Let $Q_{1}^{\prime}=Q_{1} \cap S$ and $Q_{2}^{\prime}=Q_{2}$ $\cap S$. By the Lemma there exists a prime $P_{1}$ of $S$ such that $I \not P_{1}, P_{1} \subsetneq Q_{1}^{\prime}$, and $P_{1} \subset Q_{2}^{\prime}$. Observe that $R \subset S$ has GD since $S \subset R^{\prime}$ has LO, being an integral extension. Since $P_{1} \cap R \subset P=Q_{2}^{\prime} \cap R$, it follows by GD that there is a prime $P_{2} \subset Q_{2}^{\prime}$ such that $P_{2} \cap R=P_{1} \cap R$. But this could only be true if either $P_{1}=P_{2}$ or $I \subset P_{1}$. Since both of these possibilities contradict the choice of $P_{1}$, we must conclude that $Q_{1} \cap Z\left(R^{\prime}\right)=Q_{2} \cap Z\left(R^{\prime}\right)$. Therefore, $P$ is unibranched in $R^{\prime}$.

EXAMPLE 2. Let $J_{(2)}=$ the integers localized at $(2)=2 J, R=J_{(2)}[\sqrt{-3}]=$ $\{(a+b \sqrt{-3}) / c \mid a, b, c \in J, c$ odd $\}$, and

$$
\begin{aligned}
P & =(2,1+\sqrt{-3}) \\
& =\{(a+b \sqrt{-3}) / c \mid a, b, c \in J, c \text { odd, } a \text { and } b \text { both odd or both even }\} .
\end{aligned}
$$

$\boldsymbol{R}$ is a Noetherian integral domain with unique maximal ideal $\boldsymbol{P}$. Let $\boldsymbol{u}=$ $(1+\sqrt{-3}) / 2$ and $S=R[u]=\{(a+b \sqrt{-3}) / 2 c \mid c$ odd, $a$ and $b$ both odd or both even . Since $u^{2}-u+1=0, R \subset S$ is an integral extension. Also, $P=2 S$ is the unique maximal ideal of $S$. This extension was suggested to the author by Dennis Estes.

To find a counterexample to the noncommutative version of McAdam's theorem, we need prime ideals with $\mathrm{ht}>1$. So we adjoin an indeterminate and localize again. Let

$$
S^{\prime}=S[x]_{(x, 2)}=\{p(x) / q(x) \mid p(x), q(x) \in S[x], q(0) \notin P\}
$$

and let

$$
I=(x, 2) S^{\prime}=\{p(x) / q(x) \mid p(x), q(x) \in S[x], q(0) \notin P, p(0) \in P\},
$$

the unique maximal ideal of $S^{\prime}$. Now take the subring $R^{\prime}=R[x]_{(x, 2,1+\sqrt{-3})}+I$. To see that $R^{\prime}$ is a proper subring of $S^{\prime}$, suppose $u=p(x) / q(x)+r(x) / s(x) \in R^{\prime}$ where $p(x), q(x) \in R[x] ; r(x), s(x) \in S[x] ; q(0), s(0) \notin P$; and $r(0) \in P$. Then setting $x=0$ gives $u=p(0) q(0)^{-1}+r(0) s(0)^{-1} \in R+P=R$. Since $u \in S \backslash R$, we conclude that $u \in S^{\prime} \backslash R^{\prime}$, and the subring is proper. Because $I$ is the unique 
maximal ideal of $S^{\prime}$, it follows that $S^{\prime} \backslash R^{\prime}$ consists of invertible elements. The prime ideal $I$ has ht $>1$ since $(0) \subset(x) \subset I$, and these inclusions are proper. It is straightforward to check that $S^{\prime}=R^{\prime}[u]$.

For our counterexample we now move into matrix rings. Let

$$
A=\left\{\left[\begin{array}{cc}
a u+b+e & c \\
d & a u^{-1}+b+f
\end{array}\right] \mid a, b \in R^{\prime}, c, d, e, f \in I\right\} .
$$

It is easy to check that $A$ is a ring (noting that $u^{-1}=1-u$ ). The ideal $M=$ $\left\{\left[\begin{array}{c}i \\ k\end{array}\right] \| i, j, k, l \in I\right\}$ is the unique maximal ideal of $A$. For if $C \in A \backslash M$, then $(\operatorname{det} C)^{-1} \in R^{\prime}$; so $C^{-1} \in A$. Let $B=A[u]$. Here we identify $u$ with the scalar matrix $\left[\begin{array}{ll}u & 0 \\ 0 & u\end{array}\right]$. Since $u+u^{-1}=1$ and

$$
\left[\begin{array}{cc}
u & 0 \\
0 & u^{-1}
\end{array}\right] \text { and }\left[\begin{array}{cc}
-2 u & 0 \\
0 & 0
\end{array}\right]
$$

are in $A$,

$$
\left[\begin{array}{ll}
0 & 0 \\
0 & 1
\end{array}\right]=\left[\begin{array}{ll}
u & 0 \\
0 & u
\end{array}\right]+\left[\begin{array}{cc}
u & 0 \\
0 & u^{-1}
\end{array}\right]+\left[\begin{array}{cc}
-2 u & 0 \\
0 & 0
\end{array}\right]
$$

is in $B$. Similarly, $\left[\begin{array}{ll}1 & 0 \\ 0 & 0\end{array}\right] \in B$. Using these matrix units, one can show that

$$
B=\left\{\left[\begin{array}{ll}
a & b \\
c & d
\end{array}\right] \mid a, d \in S^{\prime} ; b, c, d \in I\right\} .
$$

$B$ has two maximal ideals,

$P_{1}=\left\{\left[\begin{array}{ll}s & b \\ c & d\end{array}\right] \mid s \in S^{\prime} ; b, c, d \in I\right\} \quad$ and $\quad P_{2}=\left\{\left[\begin{array}{ll}a & b \\ c & s\end{array}\right] \mid s \in S^{\prime} ; a, b, c \in I\right\}$.

Observe that $B / P_{i} \cong S^{\prime} / I$, a field, $i=1,2$. We now make several observations regarding the central extension $A \subset B$.

1. $A \cap P_{i}=M$ and $P_{i} \cap Z(B)=\left\{\left[\begin{array}{ll}a & 0 \\ 0\end{array}\right] \mid a \in I\right\}$ for $i=1,2$. Hence, the ideal $M$ of $A$ is not weakly unibranched in $B$.

2. The extension is an integral extension of prime PI rings. Since both rings are subrings of $2 \times 2$ matrices over the commutative ring $S^{\prime}$, they satisfy the standard identity $S_{4}[6$, p. 22]. Both rings are prime since they are orders in the $2 \times 2$ matrix ring over the quotient field of $S^{\prime}$. Since $u$ is integral over $A$ and in the quotient field of $Z(A)=R^{\prime}, B$ lies in the central integral closure of $A$. It follows that the extension $A \subset B$ has GU, LO, and INC.

3. Suppose $L$ is an ideal of $B$ and $L$ has an element of the form [* $\left.{ }^{*}\right]$ with $s \in S^{\prime} \backslash I$. Then

$$
\left[\begin{array}{ll}
0 & 0 \\
0 & 1
\end{array}\right]\left[\begin{array}{cc}
* & * \\
* & s
\end{array}\right]\left[\begin{array}{ll}
0 & 0 \\
0 & 1
\end{array}\right]=\left[\begin{array}{ll}
0 & 0 \\
0 & s
\end{array}\right] \in L
$$

hence,

$$
\left[\begin{array}{cc}
0 & 0 \\
0 & s^{-1}
\end{array}\right]\left[\begin{array}{ll}
0 & 0 \\
0 & s
\end{array}\right]=\left[\begin{array}{ll}
0 & 0 \\
0 & 1
\end{array}\right] \in L
$$


It follows that $\left[\begin{array}{lll}0 & 0 \\ 0 & 1\end{array}\right]\left[\begin{array}{ll}0 & 0 \\ i & 0\end{array}\right]=\left[\begin{array}{ll}0 & 0 \\ i & 0\end{array}\right] \in L$ and $\left[\begin{array}{ll}0 & i \\ 0 & 0\end{array}\right]=\left[\begin{array}{ll}0 & i \\ 0 & 0\end{array}\right]\left[\begin{array}{ll}0 & 0 \\ 0 & 1\end{array}\right] \in L$ for all $i \in I$. If $i, j \in I$, then $\left[\begin{array}{lll}0 & i \\ 0 & 0\end{array}\right]\left[\begin{array}{ll}0 & 0 \\ j & 0\end{array}\right]=\left[\begin{array}{ll}i j & 0 \\ 0 & 0\end{array}\right] \in L$. Thus

$$
P_{2}^{2} \subset\left\{\left[\begin{array}{cc}
i & j \\
k & s
\end{array}\right] \mid i \in I^{2} ; j, k \in I ; s \in S^{\prime}\right\} \subset L .
$$

If $L$ is prime, it must be that $L=P_{2}$. Similarly, if $L$ contains an element of the form [s. $:$ ], $s \in S^{\prime} \backslash I$, and $L$ is prime, then $L=P_{1}$. So if $L$ is any prime of $B$ different from $P_{1}$ and $P_{2}$, then $L \subset\left[\begin{array}{ll}I & I \\ l & I\end{array}\right]=M=P_{1} \cap P_{2}=P_{1} \cap A=P_{2} \cap A-$ the unique maximal ideal of $A$. Therefore the extension $A \subset B$ has GD.

4. Since $R^{\prime}$ and $S^{\prime}$ are Noetherian, $A$ and $B$ are Noetherian also. The ring $A$ is integral over its center $R^{\prime}$ and ht $I>1$. It follows that the maximal ideal $M$ of $A$ has ht $>1$. By Jategaonkar's result in [2] no prime ideal of ht $>1$ can be minimal over any central element. Since $Z(A) \subset A$ has INC, $M \cap Z(A) \neq Q \cap Z(A)$ for any ht 1 prime $Q$ of $A$. Thus there must be infinitely many ht 1 primes of $A$ inside $M$. By observation 3, each of these ht 1 primes is also a prime ideal of $B$. Trivially, then, all ht 1 primes of $A$ are unibranched in $B$.

5. We see that GD does not imply that ht $>1$ prime ideals are unibranched in extensions lying within the central integral closure of a Noetherian prime PI ring. Moreover, it is possible for two primes of ht $>1$ to contain all smaller primes in their intersection. This can never happen in a commutative Noetherian ring by Krull's Principal Ideal Theorem (see, for example, the argument in [4]). As the proof of the Lemma shows, this seems to be the essential difference between the commutative and noncommutative cases.

\section{REFERENCES}

1. S. A. Amitsur and L. Small, Prime PI-rings, Bull. Amer. Math. Soc. 83 (1977), 249-251.

2. V. A. Jategaonkar, Principal ideal theorem for Noetherian P.I. rings, J. Algebra 35 (1975), 17-22.

3. Phillip Lestmann, Going down and the spec map in PI rings, Comm. Algebra G(10) (1978), 1667-1691.

4. Stephen McAdam, Going down, Duke Math. J. 39 (1972), 633-636.

5. Neal H. McCoy, A note on finite unions of ideals and subgroups, Proc. Amer. Math. Soc. 8 (1957), 633-637.

6. Claudio Procesi, Rings with polynomial identities, Dekker, New York, 1973.

7. William Schelter, Integral extensions of rings satisfying a polynomial identity, J. Algebra 40 (1976), 245-257.

Department of Mathematics, Bryan College, Dayton, Tennessee 37321 